\title{
Hierarchical fuzzy obstacle avoidance control of omnidirectional mobile robot for substation inspection
}

\author{
Li Ma \\ Kunming Power Supply Bureau, Yunnan Power Grid Co., Ltd., Kunming, Yunnan, China
}

\begin{abstract}
There are some limitations in the practical application of robot obstacle avoidance control methods. In order to realize the high-speed planning and obstacle avoidance processing of mobile robot, the path model, inspection route and path obstacles of the robot must be fully considered. Through feature extraction, the reasonable planning of substation inspection obstacle avoidance route is realized, and the control quantity set satisfying substation inspection is given. The hierarchical fuzzy obstacle avoidance control method of mobile robot is realized by using the optimal control set design. The simulation results show that the control method can solve various complex problems when the robot contour collides with obstacles, and can realize safe and fast control. The flexibility and robustness of obstacle avoidance are improved, and the motion state and speed of obstacles are also improved.
\end{abstract}

\section{Introduction}

Mobile robot is an intelligent device which integrates environmental perception, dynamic decision-making and planning, behavior control and execution. At present, the robot inspection scheme has been widely used in the theoretical application of multi-disciplinary such as automatic control and power science[1]. In the process of mobile robot inspection path planning, obstacle avoidance control has always been a difficult problem. The local unknown path planning problem is decomposed into a local unknown path planning problem. In order to solve the above problems, a hierarchical fuzzy obstacle avoidance control method for omnidirectional mobile robot for substation inspection is proposed[2]. The research shows that the method has broad application prospects and research value. According to various situations, we can judge whether it is necessary to avoid obstacles flexibly, and scientifically select the obstacle avoidance mode of mobile robot. The robot can quickly track the planning path on the premise of avoiding collision, and realize the research requirements of effective inspection of substation.

\section{Hierarchical fuzzy obstacle avoidance control for omnidirectional mobile robot}

\section{1. path planning algorithm for omnidirectional mobile robot inspection}

In order to realize the robot system, a simple and effective controller design method is provided. Based on this, the hierarchical fuzzy obstacle avoidance dynamic model of the robot is optimized to form a better robot inspection route[3], and the hierarchical fuzzy obstacle avoidance target point of the robot is set to preview whether it is consistent with the expected direction and position in the inspection process of robot man[4-6]. According to the curvature of the expected path, the appropriate step size is selected, and then the string is represented as a dotted line. Based on this, the hierarchical fuzzy obstacle avoidance dynamic structure of the robot is displayed, as shown in figure 1 .

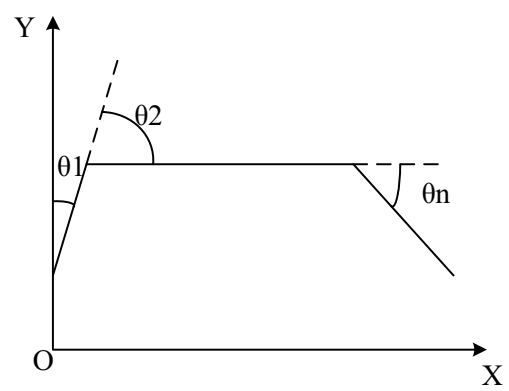

Fig. 1 Hierarchical fuzzy obstacle avoidance dynamic structure of robot

Based on the above structure, the dynamic curvature of hierarchical fuzzy obstacle avoidance of robot is further calculated, and its calculation formula is:

$$
\varphi=\theta_{n} \sum_{i=1}^{n}\left|\theta_{1}-\theta_{2}\right|^{n}
$$

In formula (1), $n$ is the angle of the first polyline once the path of the mobile robot avoids, and $\theta_{n}$ is the angle of the robot's forward route conversion; $\theta_{1}$ polyline and $\theta_{2}$ polyline represent clockwise change and counterclockwise change of route. Its absolute significance lies in that the curvature of the path will

\footnotetext{
E-mail: yueliang9112@sina.com
} 
increase regardless of changing the direction of one-way or left-right swing, that is, the bending effect will be consistent and more in line with reality. Therefore, the obstacle fixed point of the pre-robot patrol route can be determined.

$$
\Delta d=d 1-\frac{\varphi}{120^{\circ}} d 2
$$

In formula (2), d1 represents the farthest distance of the preview point, and $\mathrm{d} 2$ represents the nearest distance of the preview point. Assume that the algorithm of path selection equation is:

$$
(\mathrm{w}, \mathrm{x})+\mathrm{b}=\mathrm{w}
$$

Then further calculate the vector inner product $\mathrm{H}$ in the robot running process, and if $\mathrm{W}$ is the path vector, calculate the optimal path and the alternative path, which can be specifically written as:

$$
\begin{gathered}
H_{1}:(w, x)+b=1 \text { if } y_{i}=1 \\
H_{2}:(w \cdot x)+b=-1 \text { if } y_{i}=-1
\end{gathered}
$$

If the distance between $H_{1}$ and $H_{2}$ is called $h$, it meets the robot motion standard value of margin $=2 / \sum \mathrm{w}$, and when $h$ reaches the maximum value, it is called the best classification surface $\forall x_{i} \in X$, there are:

$$
\left\{\begin{array}{l}
\left(w \cdot x_{i}\right)+b H_{1} \ldots+1, y_{i}=1 \\
\left(w \cdot x_{i}\right)+b H_{2},-1, y_{i}=-1
\end{array}\right.
$$

Further optimization results in:

$$
\left(q_{1}-q_{2}\right)=L \theta / r t
$$

In formula (7) and (8), $r$ represents the turning radius (radius), $L$ is the turning angle determined by the robot, and the time is $t . q_{1}$ and $q_{2}$ are the angular velocities of the left and right wheels of the robot, and the constraint equations constrain the angular velocities of the two driving wheels ${ }^{[7]}$. In the coordinate constraint equation between the left and right wheels of the robot, the distance $\mathrm{L}$ between the left and right wheels is constant no matter where the robot goes. Based on trajectory coordinates, an affine nonlinear dynamic model of robot patrol particles is established.

$$
\left[\begin{array}{c}
\dot{x} \\
\dot{y} \\
\dot{\phi} \\
\dot{v} \\
\dot{\omega}
\end{array}\right]=\left[\begin{array}{c}
-v \sin (\phi) \\
v \cos (\phi) \\
\omega \\
-\frac{2 k g^{2}}{b^{3} m} v \\
-\frac{2 k g^{2} \omega^{2}}{b^{2} I} \omega
\end{array}\right]+\left[\begin{array}{c}
0 \\
0 \\
\frac{g}{b m}\left(u_{1}+u_{2}\right) \\
\frac{g \omega}{b I}\left(u_{1}-u_{2}\right)
\end{array}\right]
$$

According to the position equation $f$ of the center $O$ of two wheels in the process of robot inspection, the initial state of the robot inspection path is standardized and recorded as $[0,0,0]$, where $v$ is the speed of robot inspection and avoidance, $k$ is the constant of induced electromotive force of robot avoidance, $g$ is the speed reduction ratio of robot, $L$ represents the moment of inertia of point $O$, and 2 represents the wheelbase of driving wheel, and then the limiting parameters of robot avoidance control are calculated. The restrictions of surrounding obstacles can be expressed in the following format:

$$
g_{j}(x, y), 0
$$

In formula (9), $\mathrm{Gj}(\mathrm{x}, \mathrm{y})$ is the optimal obstacle function of the robot's inspection route. In the track coordinate system of the inspection route, the constraint conditions of obstacles in front of the robot are as follows $g_{j}: y, a x+b$. On this basis, the maximum speed control variable of robot avoidance control is obtained.

\subsection{Hierarchical fuzzy control method for omni- directional mobile robot}

Traditional manual detection methods have some problems, such as low efficiency, high risk and high labor intensity, which can not meet the requirements of current transmission line detection ${ }^{[9]}$. In order to ensure the hierarchical fuzzy obstacle avoidance effect of omnidirectional mobile robot, the inspection and control platform of omnidirectional mobile robot is standardized $^{[10]}$. The main process includes inspection and debugging, information transmission, path selection, path inspection, etc. In order to realize autonomous obstacle avoidance, it is necessary to further optimize the hierarchical fuzzy collision avoidance control method. In three aspects: fuzziness of measurement

\begin{tabular}{|c|c|c|}
\hline & $\begin{array}{l}\text { Control } \\
\text { system }\end{array}$ & $\begin{array}{c}\text { Obstacle } \\
\text { avoidance } \\
\text { information }\end{array}$ \\
\hline Get knowledge & $\begin{array}{c}\text { Expert } \\
\text { experience }\end{array}$ & $\begin{array}{l}\text { Algorithm } \\
\text { example }\end{array}$ \\
\hline $\begin{array}{l}\text { Reasoning } \\
\text { mechanism }\end{array}$ & $\begin{array}{l}\text { Heuristic } \\
\text { search }\end{array}$ & $\begin{array}{l}\text { Algorithm } \\
\text { example }\end{array}$ \\
\hline Reasoning speed & Low & High \\
\hline Fault tolerance & Low & High \\
\hline $\begin{array}{l}\text { Learning } \\
\text { mechanism }\end{array}$ & Induce & Adjust weights \\
\hline $\begin{array}{l}\text { Natural language } \\
\text { Implementation }\end{array}$ & To make clear & Not obvious \\
\hline $\begin{array}{c}\text { Natural language } \\
\text { flexibility }\end{array}$ & High & Low \\
\hline
\end{tabular}
information, reasoning mechanism and accuracy of output fuzzy sets, fuzzy logic controller is defined. In order to guarantee the obstacle avoidance accuracy of omni-directional mobile robot, it is necessary to standardize the control information of omni-directional mobile robot first. The specific contents are shown in table 1 .

With the deepening of fuzzy control technology and the expansion of its application range, the fuzzy controller has developed from a single structure to various forms. The accurate calculation of this fuzzy set is the process of transforming the fuzzy control quantity obtained by the inference engine into the output control quantity. The typical fuzzy control system is shown in 
the figure, and the concrete control structure of omni- directional mobile robot is shown in figure 2 .

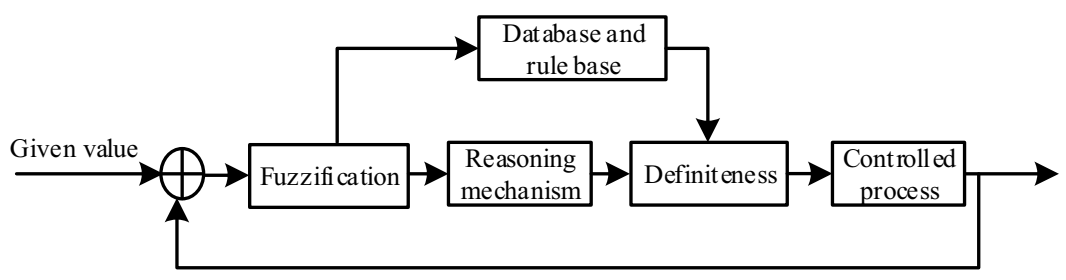

Fig. 2 Fuzzy control structure of omni-directional mobile robot

In the fuzzy control of omni-directional mobile robot, fuzzy control uses fuzzy logic to accurately detect and analyze the actual inspection route and obstacle information in the route. Fuzzy control can better resist the noise interference in the obstacle avoidance process of omni-directional mobile robot and enhance the obstacle avoidance accuracy of omni-directional mobile robot. The research shows that fuzzy control has a wide application range and a good application prospect.

\subsection{Realization of hierarchical fuzzy obstacle avoidance control for omni-directional mobile robot}

When the robot walks in a certain direction, it is easy to deviate due to many factors. In order to solve this problem, the robot collects the azimuth information of the electronic compass in real time, and corrects the azimuth error in time through the closed-loop control of the driving motor, so that the robot can realize one-way walking. Based on this, the correction steps of hierarchical fuzzy collision avoidance error for omnidirectional mobile robot are optimized as figure 3 .

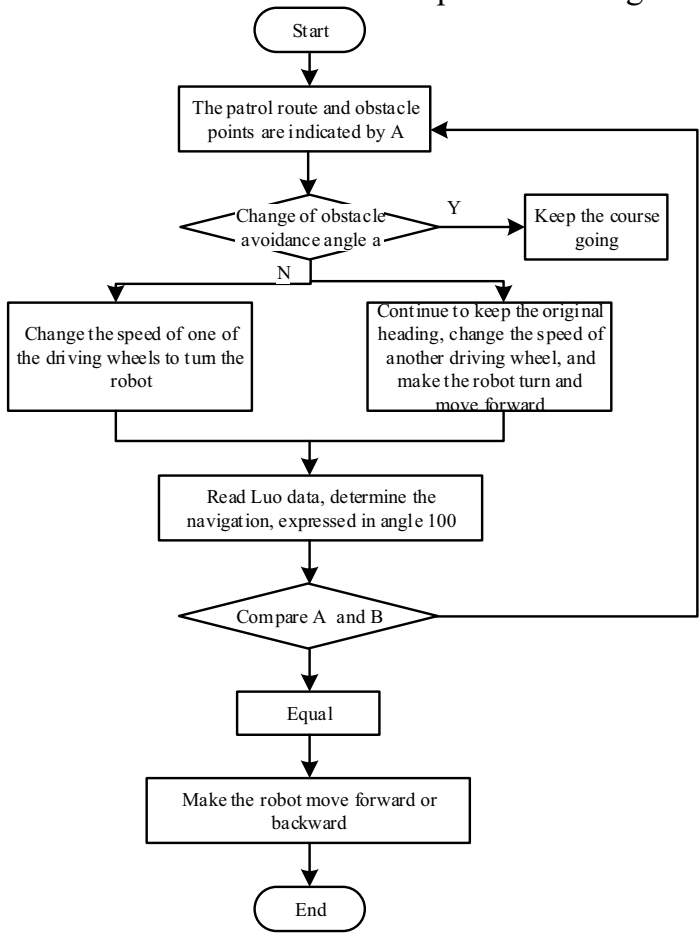

Fig. 3 the steps of modifying the collision avoidance error of mobile robot
In the process of collision avoidance, the mobile robot needs to judge the collision risk and select its obstacle avoidance behavior accordingly. All obstacles are regarded as moving objects, and whether the robot collides with the obstacles is judged by detecting the relative relationship between the historical position and the current position of the obstacles. Connect the historical position with the current position and extend to the junction with the sector. The intersection point on the fan-shaped arc can make the obstacle move away from and avoid the obstacle. So as to achieve the research requirements of accurate collision avoidance for inspection path obstacles.

\section{Analysis of experimental results}

In order to verify the effect of hierarchical fuzzy obstacle avoidance control of omnidirectional mobile robot for substation inspection, the layered fuzzy obstacle avoidance control method of omnidirectional mobile robot is simulated on MATLAB R2017a simulation system. There are a lot of motion scenes in mobile robot. In order to achieve the ideal simulation effect, in the simulation experiment, the obstacle avoidance environment of mobile robot adopts maze simulation environment, which makes it closer to the unknown real and complex environment. In reality, the feasibility and security of various obstacle avoidance algorithms need to be verified. In order to verify the feasibility and safety of the method, many tests have been carried out.

The environment outside the test is a corridor $2.5 \mathrm{~m}$ long, 7 meters wide and $2 \mathrm{~m}$ wide, separated from the test box. The size of mobile robot is $20 * 20 * 13 \mathrm{~cm}$, so the obstacles selected are $50 * 55 * 32 \mathrm{~cm}$ equipment box, $50 * 20 * 32 \mathrm{~cm}$ mechanical box, $60 * 13 * 40 \mathrm{~cm}$ test box. The obstacles selected meet the experimental requirements through the comparison between mobile robot and obstacle. The method takes the origin of environmental coordinates as the starting point to determine the location of obstacles in each scene, sends the target punctuation position through the upper computer, and conducts different positioning experiments on the obstacles of different experimental purposes. Based on the above experimental environment, the fuzzy obstacle avoidance control effect of a robot is recorded, and the specific structure is as table 2 . 
Tab. 2 collision avoidance time and error of robot controller in two groups of experiments

\begin{tabular}{ccccc}
\hline number & $\begin{array}{c}\text { traditional } \\
\text { method/min }\end{array}$ & $\begin{array}{c}\text { Collision avoidance } \\
\text { deviation/cm }\end{array}$ & $\begin{array}{c}\text { The method of this } \\
\text { paper/min }\end{array}$ & $\begin{array}{c}\text { Collision } \\
\text { avoidance } \\
\text { deviation/cm }\end{array}$ \\
\hline 1 & 24.40 & 33 & 5.34 & 7 \\
2 & 30.25 & 35 & 6.82 & 8 \\
3 & 20.42 & 39 & 4.41 & 5 \\
4 & 24.50 & 42 & 5.36 & 4 \\
\hline
\end{tabular}

It can be seen from the table that the hierarchical fuzzy obstacle avoidance control method of omnidirectional mobile robot for substation inspection proposed in this paper in the application process, the obstacle avoidance error is significantly reduced compared with the traditional method, and the hierarchical fuzzy obstacle avoidance control for substation inspection oriented omni-directional mobile robot also consumes relatively less time. However, due to the influence of many factors, omni-directional mobile robot for substation inspection is omnidirectional Hierarchical fuzzy obstacle avoidance control of robot still needs to be further improved. Furthermore, the robustness of robot obstacle avoidance control based on the traditional method and the proposed method is compared. The specific detection results are shown in figure 4.

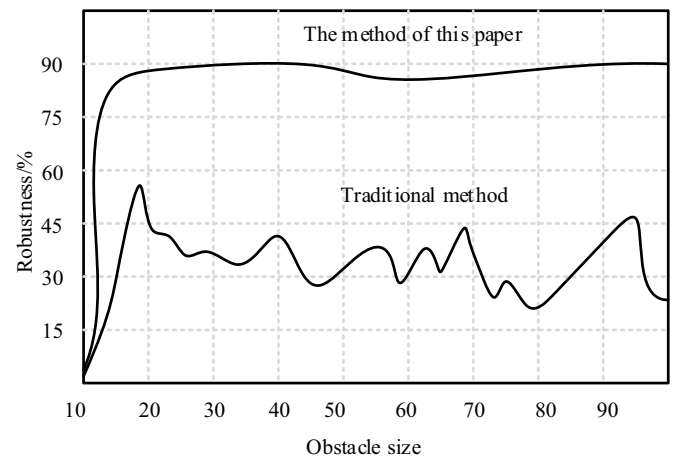

Fig. 4 comparison test results

Based on the detection results in the above figure, the hierarchical fuzzy obstacle avoidance control method for omni-directional mobile robot for substation inspection proposed in this paper is relatively more robust than the traditional control method. The experimental results show that the proposed method can achieve fast and safe obstacle avoidance. Therefore, the hierarchical fuzzy obstacle avoidance control method of omnidirectional mobile robot for substation inspection can fully meet the current research requirements.

\section{Conclusion}

According to the survival principle of omnidirectional mobile robot for substation inspection, a hierarchical fuzzy obstacle avoidance control method for omnidirectional mobile robot is proposed. Considering the complex and changeable collision problem between omni-directional mobile robot and obstacles, the constraint problem of obstacle avoidance control model of omni-directional mobile robot is optimized to realize more comprehensive obstacle avoidance control and improve work efficiency. The results show that the hierarchical fuzzy obstacle avoidance control method for omni-directional mobile robot for substation inspection is conducive to optimize the numerical calculation of obstacle avoidance control in the inspection process, and is better used for the theoretical research of robot inspection and obstacle avoidance.

\section{References}

1. Ton C., Kan Z., Mehta S. S . Obstacle avoidance control of a human-in-the-loop mobile robot system, using harmonic potential fields $[\mathrm{J}]$. Robotica, 36(4):463-483 (2018).

2. Mendes M, Coimbra A. P., Crisostomo M M . Assis - cicerone robot with visual obstacle avoidance using a stack of odometric data[J]. IAENG International Journal of Computer ence, 45(1pt.118227):219-227 (2018).

3. Wen S., Zhou N., Zhang D., et al. Variabledimensional Flower Pollination obstacle avoidance algorithm on autonomous walking of NAO robot in dynamic environment[J]. Advanced Robotics, 33(3):1-14 (2019).

4. Xie Y., Zhang Z., Wu X., et al. Obstacle Avoidance and Path Planning for Multi-Joint Manipulator in a Space Robot[J]. IEEE Access, 8(4):3511-3526 (2020).

5. Sgorbissa A. Integrated robot planning, path following, and obstacle avoidance in two and three dimensions: wheeled robots, underwater vehicles, and multicopters[J]. The International Journal of Robotics Research, 38(7):027836491984691 (2019).

6. Lee G., Chwa D. Decentralized behavior-based formation control of multiple robots considering obstacle avoidance[J]. Intelligent Service Robotics, 11(1):127-138 (2018).

7. Kun W., Bingyin R. A Method on Dynamic Path Planning for Robotic Manipulator Autonomous Obstacle Avoidance Based on an Improved RRT Algorithm[J]. Sensors, 18(2):571 (2018).

8. Ajeil F. H., Ibraheem I. K., Azar A. T., et al. Autonomous navigation and obstacle avoidance of an omnidirectional mobile robot using swarm optimization and sensors deployment[J]. International Journal of Advanced Robotic Systems, 17(3):172988142092949 (2020). 
9. Li D., Li K., Deng H., et al. The 2D Aquatic Obstacle Avoidance Control Algorithm of the Snake-Like Robot Based on Artificial Potential Field and IB-LBM[J]. Jiqiren/Robot, 40(3):346-359 (2018).

10. Sudhakara P., Ganapathy V., Priyadharshini B., et al. Obstacle Avoidance and Navigation Planning of a Wheeled Mobile Robot using Amended Artificial Potential Field Method[J]. Procedia Computer ence, 133(10):998-1004 (2018). 